\title{
Clinical efficacy of apatinib in treating metastatic gastric cancer and its effect on IL-17
}

\author{
RAN CHEN ${ }^{*}$, QI-TIAN CHEN ${ }^{*}$ and YOU-HONG DONG \\ Department of Oncology, Xiangyang No. 1 People's Hospital, \\ Huibei University of Medicine, Xiangyang, Hubei 441000, P.R. China
}

Received August 23, 2018; Accepted March 22, 2019

DOI: $10.3892 / \mathrm{ol} .2019 .10270$

\begin{abstract}
Clinical efficacy of apatinib in treating metastatic gastric cancer and its effect on the levels of serum IL-17 were investigated. A retrospective analysis was performed on 129 patients who had metastatic gastric cancer after first-line chemotherapy and were treated in Xiangyang No. 1 People's Hospital from February 2012 to February 2015. Of these patients, 78 received oral apatinib and were assigned to experimental group; and 51 received oral tegafur-gimeracil-oteracil and were assigned to control group. Clinical efficacy was compared between the two groups, and the levels of serum IL-17 were measured for all the patients. The treatment response rate in the experimental group was $52.56 \%$ and in the control group $31.37 \%$. Apparently, the treatment response rate in the experimental group was higher than that in the control group, and the difference was statistically significant $(\mathrm{P}<0.05)$. The incidence of adverse drug reactions in the experimental group was significantly lower than that in the control group $(\mathrm{P}<0.05)$. The serum level of IL-17 after one course of medication was significantly lower than that before medication in both groups $(\mathrm{P}<0.05)$. In comparison between groups, the serum level of IL-17 after one course of medication was clearly lower in the experimental group than that in the control group $(\mathrm{P}<0.05)$. Apatinib regimen was demonstrated to have less toxic side-effects in the treatment of metastatic gastric cancer than tegafur-gimeracil-oteracil regimen, indicating that apatinib has favorable safety. In addition, apatinib can downregulate IL-17 expression, which is helpful in attenuating tumor proliferation and improving the clinical efficacy. Therefore, apatinib has potential use in a wide range of clinical applications.
\end{abstract}

Correspondence to: Dr You-Hong Dong, Department of Oncology, Xiangyang No. 1 People's Hospital, Huibei University of Medicine, 15 Jiefang Road, Xiangyang, Hubei 441000, P.R. China

E-mail: dr5q55@163.com

*Contributed equally

Key words: apatinib, metastatic gastric cancer, targeted therapy, clinical efficacy, IL-17

\section{Introduction}

Gastric cancer is a common malignant tumor of the digestive system, with high incidence and complicated pathogenesis (1). Generally it has no obvious symptoms at early stages and therefore is easily ignored by patients. When diagnosed, it is often at the late stages (2). Surgery is currently the main treatment method for gastric cancer. However, for metastatic gastric cancer, the probability of postoperative recurrence is as high as $60 \%$. Therefore, medical intervention of metastatic gastric cancer is one of the current clinical challenges $(3,4)$. Chemotherapy is commonly adopted to manage metastatic gastric cancer. However, chemotherapy has some harsh and unpleasant side-effects, and its therapeutic efficacy may not be satisfactory. Many patients still die due to the recurrence of the disease in a short term (5).

Targeted therapy is a novel cancer treatment that uses specially designed drugs to target specific genes or proteins necessary for tumor growth and progression. Targeted therapy prevents cancer cells from growing and spreading, but its effect on normal cells is unknown (6). For patients the adverse reactions of targeted therapy can be more easily tolerated than those of chemotherapy. Therefore, targeted therapy for gastric cancer has drawn increasing attention in research.

Apatinib is a small-molecule tyrosine kinase inhibitor of vascular endothelial growth factor (VEGF) receptors. It is one of the popular anti-angiogenic targeting drugs which inhibits the tumor progression by blocking the formation of new blood vessels in tumor tissue (7). Apatinib has been reported to have confirmed efficacy in patients with advanced gastric cancer, but also complications, such as hypertension (8). The combination drug tegafur-gimeracil-oteracil is a combination of tegafur and two other drugs that regulate its biological effects. The synergistic action of 5-fluorouracil produced by tegafur metabolism, gimeracil that inhibits 5-fluorouracil degradation, and oteracil that modulates 5-fluorouracil distribution produces a good anticancer effect. This combination drug is widely used in the treatment of gastric cancer as a first-line drug due to its efficacy, safety and convenience (9).

A previous study (10) has indicated that one of the important mechanisms for the occurrence and development of gastric cancer is the presence of chronic inflammation. IL-17 is a pro-inflammatory factor. According to literature, IL-17 not only plays a role in autoimmune diseases and in the defense 
against pathogenic microbes, but also promotes tumor angiogenesis and plays an important role in tumorigenesis, growth and metastasis (11).

Both apatinib and IL-17 are associated with tumor angiogenesis, but the effect of apatinib on IL-17 expression has rarely been reported. In this study, the clinical efficacy of apatinib was compared with that of tegafur-gimeracil-oteracil in treating metastatic gastric cancer, and their effects on the levels of serum IL-17 were also evaluated. The aim was to provide an optimal regimen for treating patients with metastatic gastric cancer.

\section{Patients and methods}

Patients. A retrospective analysis was performed on 129 patients who had metastatic gastric cancer after first-line chemotherapy and were treated in Xiangyang No.1 People's Hospital (Xiangyang, China) from February 2012 to February 2015. Sixty-seven males and 62 females, with an average age of $60.2 \pm 5.7$ years, were enrolled. Of these patients, 78 received oral apatinib and were assigned to the experimental group and 51 received oral tegafur-gimeracil-oteracil and were assigned to the control group. As shown in Table I, there were no statistically significant differences in sex, age, BMI, drinking status, tumor location and metastatic sites between the two groups $(\mathrm{P}>0.05)$.

Inclusion and exclusion criteria. Patients diagnosed by pathological tests with advanced gastric cancer with metastases and patients who underwent IL-17 test were included. Patients who met the following criteria were excluded from this study: i) patients who had other serious organ diseases; ii) who were not cooperative with the examination; and iii) with cognitive-communication disorders. Patients who participated in this research had complete clinical data. All patients and their families voluntarily participated in this study and signed an informed consent. The study was approved by the Ethics Committee of Xiangyang No. 1 People's Hospital.

Experimental drugs and reagents. Apatinib (SFDA approval no. H20140105) and tegafur-gimeracil-oteracil (SFDA approval no.H20100135) were purchased from Jiangsu Hengrui Medicine Co., Ltd. (Lianyungang, China). IL-17 ELISA kit was purchased from Shenzhen Juying Biotechnology Co., Ltd. (Shenzhen, China).

Experimental methods. Patients in the experimental group received oral apatinib, $850 \mathrm{mg}$ once daily, half an hour after a meal. Each cycle consisted of 4 weeks of treatment. Patients in the control group received oral tegafur-gimeracil-oteracil, $40 \mathrm{mg} / \mathrm{m}^{2}$ twice daily. Each cycle consisted of 3 weeks of treatment, and one course of treatment consisted of 3 cycles. The liver and kidney function of all patients was regularly checked, and blood routine examinations were regularly performed. When a patient had a serious adverse reaction, appropriate treatment was provided, and the medication was discontinued or the dose was reduced by half. Venous blood samples were drawn from fasting patients of the two groups, before treatment and after one course of treatment. Serum was collected after centrifugation at $3,000 \times \mathrm{g}$ for $15 \mathrm{~min}$
Table I. General information of patients [n (\%)].

\begin{tabular}{|c|c|c|c|c|}
\hline Variable & $\begin{array}{c}\text { Experimental } \\
\text { group, } \mathrm{n}=78\end{array}$ & $\begin{array}{c}\text { Control } \\
\text { group, } n=51\end{array}$ & $\chi^{2}$ value & $\mathrm{P}$-value \\
\hline \multicolumn{5}{|l|}{ Sex } \\
\hline Male & $40(51.28)$ & 27 (52.94) & 0.034 & 0.854 \\
\hline Female & $38(48.72)$ & $24(47.06)$ & & \\
\hline \multicolumn{5}{|l|}{ Age (years) } \\
\hline$\leq 60$ & $41(52.56)$ & $28(54.90)$ & 0.068 & 0.795 \\
\hline$>60$ & $37(47.44)$ & $23(45.10)$ & & \\
\hline \multicolumn{5}{|l|}{ BMI $\left(\mathrm{kg} / \mathrm{m}^{2}\right)$} \\
\hline$\leq 22$ & $33(42.31)$ & $22(43.14)$ & 0.009 & 0.926 \\
\hline$>22$ & 45 (57.69) & $29(56.86)$ & & \\
\hline \multicolumn{5}{|l|}{ Drinking } \\
\hline Yes & $51(65.38)$ & $33(64.71)$ & 0.006 & 0.937 \\
\hline No & 27 (34.62) & $18(35.29)$ & & \\
\hline \multicolumn{5}{|c|}{ Tumor location } \\
\hline Fundus & 24 (30.77) & $16(31.37)$ & 0.107 & 0.948 \\
\hline Corpus & $25(32.05)$ & $15(29.41)$ & & \\
\hline Antrum & $29(37.18)$ & $20(39.22)$ & & \\
\hline \multicolumn{5}{|c|}{ Metastatic sites } \\
\hline Liver & $32(41.03)$ & $21(41.18)$ & 0.090 & 0.956 \\
\hline Peritoneum & $26(33.33)$ & $18(35.29)$ & & \\
\hline Lung & $20(25.64)$ & $12(23.53)$ & & \\
\hline
\end{tabular}

at $4^{\circ} \mathrm{C}$. The levels of serum IL-17 before and after treatment were measured in strict accordance with the manufacturer's instructions of the ELISA kit.

Observed indicators. The incidence of adverse reactions after one course of treatment was statistically analyzed. Adverse reactions included hypertension, proteinuria, hand-foot syndrome, myelosuppression and diarrhea. The clinical efficacy was evaluated and assigned as complete remission, partial remission, stable disease and progressive disease, according to the RECIST 1.1 criteria (12). Efficiency = (number of complete remission + number of partial remission)/(total number of patients) $\times 100 \%$. The progression-free survival time and the 3 -year overall survival rate were recorded and compared between the two groups.

Statistical analysis. SPSS 18.0 software [Bizinsight (Beijing) Information Technology Co. Ltd., Beijing, China] was used for the statistical analysis of the experimental data. Figures were generated using the GraphPad Prism 6 software (GraphPad Software, Inc., La Jolla, CA, USA). The $\chi^{2}$ test was used for enumeration data. Measurement data were expressed as mean \pm standard deviation. t-test was used for their comparison between groups and paired t-test was used in comparison within groups before and after treatment. Analysis of variance was used for the comparison among groups and LSD was the post hoc test. Kaplan-Meier survival analysis was used to generate the survival curves and long-rank test was used for their comparison. $\mathrm{P}<0.05$ was considered to indicate statistically significant difference. 
Table II. Changes of serum IL-17 in patients of the two groups before and after treatment.

\begin{tabular}{lccr}
\hline Group & Before medication & After one course of medication & t value \\
\hline Experimental group, $\mathrm{n}=78$ & $8.81 \pm 2.36$ & $5.33 \pm 2.19$ & 9.546 \\
Control group, $\mathrm{n}=51$ & $8.87 \pm 2.29$ & $6.63 \pm 2.21$ & 5.027 \\
t value & 0.143 & 3.285 & - \\
P-value & 0.887 & $<0.050$ & - \\
\hline
\end{tabular}

Table III. Incidence of adverse reactions [n (\%)].

\begin{tabular}{|c|c|c|c|c|}
\hline Adverse reaction & Experimental group, $\mathrm{n}=78$ & Control group, $\mathrm{n}=51$ & $\chi^{2}$ value & P-value \\
\hline Hypertension & $3(3.85)$ & $5(9.80)$ & - & - \\
\hline Proteinuria & $1(1.28)$ & $3(5.88)$ & - & - \\
\hline Hand-foot syndrome & $2(2.56)$ & $4(7.84)$ & - & - \\
\hline Myelosuppression & $1(1.28)$ & $3(5.88)$ & - & - \\
\hline Diarrhea & $1(1.28)$ & $3(5.88)$ & - & - \\
\hline Total incidence & $8(10.26)$ & $18(35.29)$ & 12.01 & $<0.05$ \\
\hline
\end{tabular}

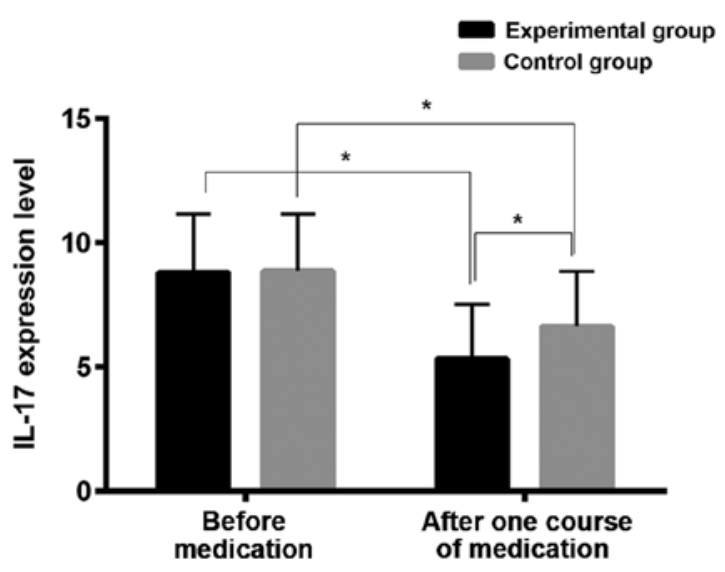

Figure 1. Changes of serum IL-17 in patients of the two groups before and after treatment. ELISA results showed that the levels of serum IL-17 after one course of medication were significantly lower than that before medication in both the experimental and the control group ( $\mathrm{P}<0.05)$. In comparison between groups, the level of serum IL-17 in the experimental group was clearly lower than that in the control group after one course of medication $(" \mathrm{P}<0.05)$.

\section{Results}

Changes of serum IL-17 in patients of the two groups before and after treatment. As shown in Table II and Fig. 1, the serum levels of IL-17 after one course of medication were significantly lower than that before medication in both the experimental and the control group $(\mathrm{P}<0.05)$. In comparison between groups, after one course of medication the serum level of IL-17 in the experimental group was clearly lower than that in the control group $(\mathrm{P}<0.05)$.

Comparison of the incidence of adverse reactions between the two groups. As shown in Table III, the number of patients who developed hypertension, proteinuria, hand-foot syndrome, myelosuppression and diarrhea were $3,1,2,1$, and 1 , respectively, in the experimental group, and 5, 3, 4, 3, and 3, respectively, in the control group. The incidence of adverse reactions in the experimental group was $10.26 \%$, which was significantly lower than that in the control group (35.29\%). The difference was statistically significant $(\mathrm{P}<0.05)$.

Comparison of the short-term efficacy between the two groups. As shown in Table IV and Fig. 2, the treatment response rate in the experimental group was $52.56 \%$, which was significantly higher than that in the control group (31.37\%). The difference was statistically significant $(\mathrm{P}<0.05)$.

Comparison of the progression-free survival time and the 3-year overall survival rate between the two groups. The progression-free survival time of patients in the experimental group was $1.23 \pm 0.23$ years, and the 3 -year overall survival rate was $44.87 \%$. In the control group the progression-free survival time was $0.74 \pm 0.12$ years, and the 3 -year overall survival rate was $19.61 \%$. Both the progression-free survival time and the 3-year overall survival rate in the experimental group were significantly higher than those in the control group $(\mathrm{P}<0.001)$ (Table V and Fig. 3).

\section{Discussion}

At present, dietary habits and lifestyle are changing with the rapid development of society. These changes have caused an increase of the incidence of digestive tract cancers (13). Gastric cancer is one of the common digestive tract tumors, which is among the top ranking cancers in terms of cancer-related deaths (14). Due to no obvious symptoms at the early stages of gastric cancer, many patients miss the chance of surgical treatment when their cancer is finally discovered. After chemotherapy, a high incidence of metastasis is observed in patients 
Table IV. Comparison of short-term efficacy between the two groups [n (\%)].

\begin{tabular}{lcccr}
\hline Efficacy & Experimental group, $\mathrm{n}=78$ & Control group, $\mathrm{n}=51$ & $\chi^{2}$ value & P-value \\
\hline Complete remission & 0 & 0 & - & - \\
Partial remission & $41(52.56)$ & $16(31.37)$ & 5.615 & $<0.050$ \\
Stable disease & $20(25.64)$ & $15(29.41)$ & 0.222 & 0.638 \\
Progressive disease & $17(21.79)$ & $20(39.22)$ & 4.575 & 0.032 \\
Response rate & $41(52.56)$ & $16(31.37)$ & 5.615 & $<0.050$ \\
\hline
\end{tabular}

Table V. Progression-free survival time and 3-year overall survival rate of patients in the two groups.

\begin{tabular}{lcccr}
\hline Items & Experimental group, $\mathrm{n}=78$ & Control group, $\mathrm{n}=51$ & $\mathrm{t} / \chi^{2}$ value & P-value \\
\hline Progression-free survival time (years) & $1.23 \pm 0.23$ & $0.74 \pm 0.12$ & 14.01 & $<0.001$ \\
3-year overall survival rate & $35(44.87)$ & $10(19.61)$ & 8.665 & $<0.001$ \\
\hline
\end{tabular}

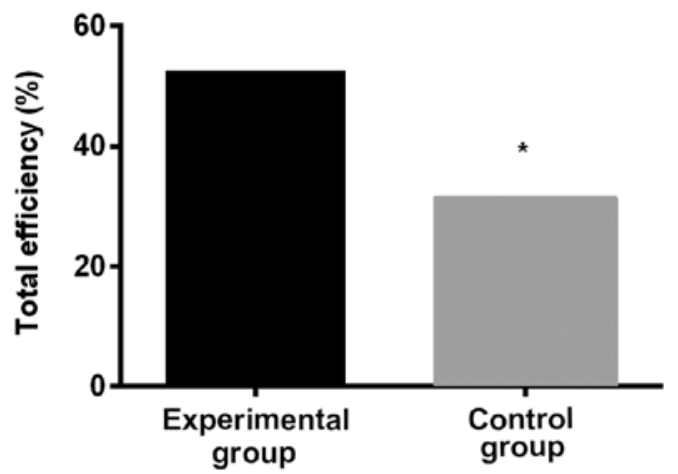

Figure 2. Treatment response rates in the two groups. The response rate in the experimental group was significantly higher than that in the control group $\left({ }^{*} \mathrm{P}<0.05\right)$.

with advanced gastric cancer, resulting in a very low 5-year survival rate for most patients (15). Chemotherapy-based comprehensive regimen is currently widely used in the treatment of metastatic gastric cancer. However, the prognosis of patients with metastatic gastric cancer is still very poor after treatment (16). Apatinib is a small-molecule targeted antitumor drug. It inhibits tumor angiogenesis which is a key step in tumor growth and metastasis (17). The signaling pathway mediated by VEGF and its receptor (VEGFR) is involved in tumor angiogenesis and plays an important role in the process. Apatinib acts by competing with VEGF for the ATP-binding site of VEGFR-2 in cells. The resulting inhibition of the VEGF-VEGFR pathway prevents VEGFR-2 autophosphorylation and blocks downstream signaling. Overall, apatinib produces a powerful antitumor effect by inhibiting the formation of new blood vessels in tumor tissue (18). IL-17 is a key cytokine that mediates innate and adaptive immunity. It is mainly secreted by Th17 cells (19). According to literature, IL-17 plays an important role in the onset and progression of gastric cancer (20). Zhang et al have reported that the IL-17 level in peripheral blood of patients with advanced gastric cancer is higher than that in patients with early gastric

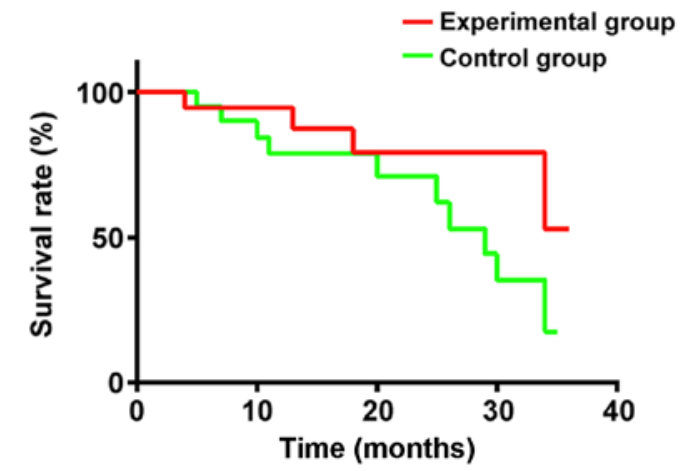

Figure 3. Comparison of the 3-year overall survival rate between the two groups. The 3-year overall survival rate of the experimental group was significantly higher than that of the control group $(\mathrm{P}<0.001)$.

cancer (21). Therefore, it is postulated that the overexpression of IL-17 may be associated with the progression of gastric cancer. Ma et al have reported that the serum level of IL-17 in patients with gastric cancer is higher than that in healthy people (22). The underlying reason is that the activation of the signal transduction and activation of transcription-3 pathway by IL-6 induces the expression of the Th17-specific transcription factor orphan nuclear receptor $\gamma t$. The orphan nuclear receptor $\gamma \mathrm{t}$ promotes the differentiation of primary $\mathrm{T}$ cells into Th17 cells, which ultimately leads to overexpression of IL-17.

In this study, the clinical efficacy of apatinib and tegafur-gimeracil-oteracil in the treatment of metastatic gastric cancer was compared, and their effect on the serum levels of IL-17 was also explored. The results showed that the serum level of IL-17 was significantly lower in the experimental group after one course of apatinib medication, compared to control group $(\mathrm{P}<0.05)$. Up to our knowledge, the effect of apatinib on the serum level of IL-17 in patients with metastatic gastric cancer is reported for the first time. It has been postulated that apatinib-induced downregulation of IL-17 is a result of inhibition of tumor angiogenesis in which IL-17 is involved. In terms of incidence of adverse reactions between the two groups, our 
results showed that the overall incidence of adverse reactions was $10.26 \%$ in the experimental group, which was significantly lower than $35.29 \%$ in the control group. The difference was statistically significant $(\mathrm{P}<0.05)$. Scott et al have reported that apatinib has higher bio-availability, more favorable safety and higher adverse reaction tolerance profile than traditional gastric cancer drugs (23). This is consistent with our findings. In comparison of clinical efficacy between the two groups, the results showed that the treatment response rate in the experimental group was significantly higher than that in the control group $(\mathrm{P}<0.05)$. Currently, there are few clinical studies on apatinib. Geng and Li have reported that apatinib demonstrates better clinical efficacy in patients with advanced gastric cancer than tegafur-gimeracil-oteracil (24). We compared the progression-free survival time and 3-year overall survival rate of patients in the two groups, and the results showed that in the experimental group, the progression-free survival time was significantly longer and the 3-year overall survival rate was significantly higher than those in the control group. In the study reported by Wainberg et al (25) at the 2014 ASCO meeting, the results revealed that the overall survival time of patients with metastatic gastric cancer after taking apatinib was higher than those using placebo, and all patients included in the study had failed second-line chemotherapy. This also verifies our conclusions.

In summary, compared with the traditional drug tegafur-gimeracil-oteracil, apatinib is safer with milder adverse effects in treating patients with metastatic gastric cancer. In addition, apatinib can attenuate the expression of IL-17 in the serum of patients with gastric cancer. Therefore, apatinib has potential use in a wide range of clinical applications due to its excellent efficacy in treating patients with metastatic gastric cancer. However, it is undeniable that the small sample size in this study could result in bias in findings. Therefore, studies with larger sample size are needed for verification.

\section{Acknowledgements}

Not applicable.

\section{Funding}

No funding was received.

\section{Availability of data and materials}

The datasets used and/or analyzed during the current study are available from the corresponding author on reasonable request.

\section{Authors' contributions}

RC and YHD were responsible for the treatment of patients. QTC and YHD collected and analyzed the general data of patients. RC drafted the manuscript. All authors read and approved the final manuscript.

\section{Ethics approval and consent to participate}

This study was approved by the Ethics Committee of Xiangyang No. 1 People's Hospital (Xiangyang, China).
Patients who participated in this research had complete clinical data. Signed informed consents were obtained from the patients or their guardians.

\section{Patient consent for publication}

Not applicable.

\section{Competing interests}

The authors declare that they have no competing interests.

\section{References}

1. Amieva M and Peek RM Jr: Pathobiology of Helicobacter pylori-induced gastric cancer. Gastroenterology 150: 64-78, 2016.

2. Guilford PJ: Markers for detection of gastric cancer: US Patent, US 9,310,370 B2. Filed May 14, 2010; issued April 12, 2016.

3. Liang H: Intraperitoneal chemotherapy for locally advanced gastric cancer to prevent and treat peritoneal carcinomatosis. Transl Gastroenterol Hepatol 1: 62, 2016.

4. Mikami J,Kimura Y, Makari Y, Sawada G, Nakahira S, Nakata K, Yamamoto T, Tsujie M and Ohsato $\mathrm{H}$ : A case of self-expandable metallic stent placement to treat colon obstruction due to metastatic gastric cancer. Gan To Kagaku Ryoho 43: 1899-1901, 2016 (In Japanese).

5. Ghosn M, Tabchi S, Kourie HR and Tehfe M: Metastatic gastric cancer treatment: Second line and beyond. World J Gastroenterol 22: 3069-3077, 2016

6. Hofmann F, Marconi LS, Stewart F, Lam TB, Bex A, Canfield SE and Ljungberg B: Targeted therapy for metastatic renal cell carcinoma (protocol). Cochrane Database Syst Rev 9: CD012796, 2017.

7. Li J, Zhao X, Chen L, Guo H, Lv F, Jia K, Yv K, Wang F, Li C, Qian J, et al: Safety and pharmacokinetics of novel selective vascular endothelial growth factor receptor-2 inhibitor YN968D1 in patients with advanced malignancies. BMC Cancer 10: 529, 2010.

8. Li J, Qin S, Xu J, Guo W, Xiong J, Bai Y, Sun G, Yang Y, Wang L, Xu N, et al: Apatinib for chemotherapy-refractory advanced metastatic gastric cancer: Results from a randomized, placebo-controlled, parallel-arm, phase II trial. J Clin Oncol 31: 3219-3225, 2013

9. Shen Q, Ma X, Hu W, Chen L, Huang J and Guo Y: Intensity-modulated radiotherapy versus three-dimensional conformal radiotherapy for stage I-II natural killer/T-cell lymphoma nasal type: Dosimetric and clinical results. Radiat Oncol 8: 152, 2013.

10. Wang N, Yang J, Lu J, Qiao Q, Bao G, Wu T and He X: IL-17 gene polymorphism is associated with susceptibility to gastric cancer. Tumour Biol 35: 10025-10030, 2014.

11. Maniati E, Soper R and Hagemann T: Up for mischief? IL-17/Th17 in the tumour microenvironment. Oncogene 29: 5653-5662, 2010.

12. Nishiofuku H, Tanaka T, Sakaguchi H, Yamamoto K, Inoue M, Sueyoshi S, Shinnkai T, Hasegawa M and Kichikawa K: Hepatic arterial infusion chemotherapy combined with venous embolization in a patient with hepatic metastases with an arteriovenous shunt. Cardiovasc Intervent Radiol 32: 796-800, 2009.

13. Thomas RM and Sobin LH: Gastrointestinal cancer. Cancer 75 (Suppl): 154-170, 1995.

14. Yoshida K, Yamaguchi K, Okumura N, Tanahashi T and Kodera Y: Is conversion therapy possible in stage IV gastric cancer: The proposal of new biological categories of classification. Gastric Cancer 19: 329-338, 2016.

15. Hsu PI, Chen CH, Hsieh CS, Chang WC, Lai KH, Lo GH, Hsu PN, Tsay FW, Chen YS, Hsiao M, et al: Alpha1-antitrypsin precursor in gastric juice is a novel biomarker for gastric cancer and ulcer. Clin Cancer Res 13: 876-883, 2007.

16. Carter GC, Kaltenboeck A, Ivanova J, Liepa AM, San Roman A, Koh M, Rajan N, Cheng R, Birnbaum HG, Kim JS, et al: Real-world treatment patterns among patients with advanced gastric cancer in South Korea. Cancer Res Treat 49: 578-587, 2017.

17. Roviello G, Ravelli A, Polom K, Petrioli R, Marano L, Marrelli D, Roviello F and Generali D: Apatinib: A novel receptor tyrosine kinase inhibitor for the treatment of gastric cancer. Cancer Lett 372: 187-191, 2016. 
18. Zhang H: Apatinib for molecular targeted therapy in tumor. Drug Des Devel Ther 9: 6075-6081, 2015.

19. O'Brien RL, Roark CL and Born WK: IL-17-producing gammadelta T cells. Eur J Immunol 39: 662-666, 2009.

20. Martin-Orozco N and Dong C: The IL-17/IL-23 axis of inflammation in cancer: Friend or foe? Curr Opin Investig Drugs 10: 543-549, 2009.

21. Zhang B, Rong G, Wei H, Zhang M, Bi J, Ma L, Xue X, Wei G, Liu X and Fang G: The prevalence of Th17 cells in patients with gastric cancer. Biochem Biophys Res Commun 374: 533-537, 2008.

22. Ma D, Zhu X, Zhao P, Zhao C, Li X, Zhu Y, Li L, Sun J, Peng J, Ji C and Hou M: Profile of Th17 cytokines (IL-17, TGF-beta, IL-6) and Th1 cytokine (IFN-gamma) in patients with immune thrombocytopenic purpura. Ann Hematol 87: 899-904, 2008.
23. Scott AJ, Messersmith WA and Jimeno A: Apatinib: A promising oral antiangiogenic agent in the treatment of multiple solid tumors. Drugs Today (Barc) 51: 223-229, 2015.

24. Geng R and Li J: Apatinib for the treatment of gastric cancer. Expert Opin Pharmacother 16: 117-122, 2015.

25. Wainberg ZA, Anghel A, Desai AJ, Ayala R, Luo T, Safran B, Fejzo MS, Hecht JR, Slamon DJ and Finn RS: Lapatinib, a dual EGFR and HER2 kinase inhibitor, selectively inhibits HER2-amplified human gastric cancer cells and is synergistic with trastuzumab in vitro and in vivo. Clin Cancer Res 16: 1509-1519, 2010

This work is licensed under a Creative Commons

Attribution-NonCommercial-NoDerivatives 4.0 International (CC BY-NC-ND 4.0) License. 